\title{
Extrinsic Influence Factors in Software Reliability: A Study of 200,000 Windows Machines
}

\author{
Christian Bird * Venkatesh-Prasad Ranganath" Thomas Zimmermann * \\ Nachiappan Nagappan * Andreas Zeller ${ }^{+}$ \\ * Microsoft Research \\ Washington, USA \\ \{cbird·tzimmer·nachin\}@microsoft.com \\ Kansas State University \\ Kansas, USA \\ rvprasad@ksu.edu \\ + Saarland University \\ Saarbrücken, Germany \\ zeller@cs.uni-saarland.de
}

\begin{abstract}
Reliability of software depends not only on intrinsic factors such as its code properties, but also on extrinsic factors - that is, the properties of the environment it operates in. In an empirical study of more than 200,000 Windows users, we found that the reliability of individual applications is related to whether and which other applications are installed: While games and file-sharing applications tend to decrease the reliability of other applications, security applications tend to increase it. Furthermore, application reliability is related to the usage profiles of these applications; generally, the more an application is used, the more likely it is to have negative impact on reliability of others. As a consequence, software testers must be careful to investigate and control these factors
\end{abstract}

\section{Categories and Subject Descriptors}

D.2.4 [Software Engineering]: Software/Program Verification - reliability, statistical methods; D.4.5 [Operating Systems]: Reliability; C.4 [Performance of Systems] - measurement techniques; reliability, availability, and serviceability; D.2.5 [Software Engineering]: Testing and Debugging - monitors, tracing;

\section{General Terms}

Reliability, Measurement

\section{Keywords}

Windows

\section{INTRODUCTION}

When assessing the reliability of an application, for instance during in-house testing, one must make reasonable assumptions on what can and what cannot happen in the environment. For instance, a new application would be tested on a specific set of hardware and operating system configurations. As the operating system shields applications from influencing each other directly, one might assume that it should not matter whether other applications are installed or being used. But does this assumption hold?

As a computer user, you may have experienced applications being incompatible with each other. If you install the popular Steam games platform on your Windows PC, for instance, there are no less than 75 other applications known to cause issues with Steam and playing games [1]. The list includes popular anti-virus software such as

Permission to make digital or hard copies of all or part of this work for personal or classroom use is granted without fee provided that copies are not made or distributed for profit or commercial advantage and that copies bear this notice and the full citation on the first page. To copy otherwise, or republish, to post on servers or to redistribute to lists, requires prior specific permission and/or a fee.

ICSE'14, May 31 - June 7, 2014, Hyderabad, India.

Copyright 2014 ACM 978-1-4503-2768-8/14/05 ... \$15.00
BitDefender, communication software such as Skype, or backup software such as Western Digital Backup Anywhere. For six out of the 75 applications, Steam support recommends that they "be fully uninstalled from your system if disabling them does not resolve the issue."

Such incompatibilities are not uncommon: When installing Kaspersky Anti-Virus software, it automatically uninstalls incompatible products (most of these being other anti-virus programs) from a list of 593 programs; another 173 are deleted after obtaining user confirmation [2]. Clearly, some programs are not meant to peacefully co-exist on your system.

In this paper we investigate how the environment of a software application determines its reliability. We address questions such as: How frequent are such incompatibilities between applications? How would an application's reliability be affected by its environmentbe it other applications, the system configuration, or hardware? What do such influences imply as it comes to assessing software reliability?

To answer these questions, we analyzed reliability data from more than 200,000 users of Microsoft Windows and associated crashes of the most frequently used applications with software and hardware features. We introduce and use a method of data analysis that we term "Influence Factors" - a general pattern that untangles the influences of many factors on many different outcomes that we describe in detail in Section 3.5. This pattern enabled us to determine which software and hardware configurations are the most failure-prone; and which extrinsic factors (factors in the environment) determine the reliability of individual applications. Our key findings include:

- A lower reliability of an application can be related to simply using another single application;

- Usage of file-sharing programs universally correlates with lower application reliability;

- The mere installation of one application may affect the reliability of others:

- While most security applications increase reliability, there are also ones that correlate with decreased reliability; and

- Less powerful hardware correlates with lower application reliability.

In practice, this means that even the best-written software may fail due to interference with other applications; and therefore, users do and will experience failures for which no single vendor is responsible. This alone may not come as a surprise to computer users. However, to our knowledge, this study is the first to analyze the problem and to quantify its extent.

Our findings have a number of consequences for future research and development, ranging from inconvenient to challenging: 
- Application testers cannot simply test on out-of-the-box systems (which is the standard practice). Instead, they must test on environments with several applications that could possibly interfere. With the permission of users, they must collect and aggregate usage and failure data, and use an analysis such as ours to detect interference.

- Application designers must anticipate and handle issues stemming from interference with third party applications. In essence, we need mechanisms to express expected interaction, and to detect and prevent unexpected interaction.

- Operating systems of the future will need much better organized ecosystems, with clear, enforceable rules such that applications can better coexist. How do we specify this? Who enforces the rules? Can we keep systems flexible and reliable at the same time? Who will be in charge of the computer - vendors or users? Moving and enforcing specifications and architectural rules from applications to the system level brings plenty of challenges for research.

The remainder of the paper is organized as follows: Section 2 discusses our data source. Section 3 presents the most frequently used applications, their features, and their categorization and discusses the study design, based on logistic regression and frequent itemset mining; it also generalizes the study setting to the general "Influence Factors" data analysis pattern. Section 4 discusses the influence of hardware and software features on reliability of Windows and applications. Section 5 presents the impact that specific combinations of hardware and/or application features have on reliability. Section 6 discusses our results in detail, including threats to validity; Section 7 discusses related work and Section 8 closes with conclusions and consequences.

\section{THE WINDOWS CUSTOMER EXPERI- ENCE IMPROVEMENT PROGRAM (CEIP)}

When first starting a new installation of Windows 7, customers can opt to participate in the Windows Customer Experience Improvement Program (CEIP), a program to collect information about computer hardware and operating system usage. When the program is activated, the computer will automatically collect information on the system configuration and software reliability, including

- Hardware configuration — such as the number of processors, processor speed, screen resolution, or graphics card;

- Software usage and reliability-i.e., which binaries were started, whether they terminated normally, crashed or hung.

When the PC is connected to the Internet via a broadband connection, this data is securely sent to servers in Microsoft-controlled facilities, and made available to the Windows team [3]. CEIP was designed with customer privacy in mind and goes to great lengths to preserve such privacy. As one such measure, CEIP randomly generates a number called a globally unique identifier (GUID) that is sent to Microsoft with every CEIP report. The GUID lets Microsoft determine which data is sent from a particular machine over time. However, the GUID does not contain any personal information and is not used to identify anyone.

In this study, our goal was to learn how system and application reliability would be affected by the extrinsic factors listed above. We therefore accessed a slice of CEIP data collected in the period of January to March, 2011, representing configuration, usage, and reliability information of more than 200,000 individual machines whose users opted into the CEIP.
The CEIP attempts to relate reliability to usage profiles - that is, how the computer is actually used. For this purpose, it tracks the following information:

- Binary launches. For each binary (an individual executable file launched by the operating system), the CEIP tracks when and how often it was launched.

- Binary meta-data. For each binary, the CEIP tracks meta-data such as file name, program name, or vendor name.

- Binary crashes. The CEIP records the number of normal and abnormal terminations for each binary.

We use application crashes (abnormal terminations) as our measure of reliability. From the CEIP data, one can see how frequently, for example, a specific image manipulation application and a specific antivirus application were launched, and how frequently they crashed. Data, files, settings or any other information accessed or produced by these programs is not collected, however. We can thus characterize usage only by the programs launched.

\section{METHODOLOGY}

We first describe how we identified and categorized the applications that we analyzed in our study (Section 3.1), the features that we use to model reliability (Section 3.2) and our analysis methods based on logistic regression (Section 3.3), influence networks (Section 3.4), and frequent itemset mining (Section 3.5).

\subsection{Identification and Selection of Applications}

Software application typically consists of multiple binaries. These binaries and applications also come in multiple versions; there may even be variants of binaries for specific locales or hardware configurations. Our first task thus was to map binaries to applications. For this purpose, for each binary, we identified the application it was part of from the attached meta-data. All binaries related to, for example Skype, would thus be mapped to a single application.

In order to limit the size of our study, we focused on the most frequently used applications. Aiming for the top 50 applications, we collected data for the top 55, out of which two were found to be duplicates. We retained 53 applications, which we grouped into seven categories. The functionality of each application was the primary attribute used for categorization.

- 14 internet applications. This category contained Internet browsers, e-mail as well as instant messaging programs.

- 5 file applications. This included popular file sharing programs as well as unarchivers.

- 5 office applications. This included "classic" productivity applications such as word processors, spreadsheet systems, and presentation programs.

- 3 photography applications. This category contained programs to manage and manipulate digital photographs.

- 11 security applications. This includes anti-virus software as well as firewall and other network protection software.

- 3 games. All are graphics-intensive and highly interactive.

- 12 media applications. This category included software for managing, playing, and streaming music and video files.

From an experimental design perspective, the categorization was initially performed by the last author and all researchers reviewed and agreed on the final categorization used in this paper. As this categorization is an important attributed of this study, we took additional steps to validate that the categorization was as objective as possible. We measured inter-rater agreement for the categorization with three additional, independent raters (who are not co-authors of this paper); 
the Fleiss' Kappa [4] values between the external raters and our categorization were $0.679,0.736$, and 0.815 (all values statistically significant at $\mathrm{p}<0.001$ ), which can be considered a substantial agreement [5].

These categories also reflect what the PCs were mainly used for: Browsing the web, playing games, office work, managing music, photos, or videos - and sharing and downloading files. (We did not find a single programming or scientific application in the most used applications.) The proportions of the categories do not reflect the proportions of usage, though; it is just that there are many more games than office applications; hence, for a single game, it is more difficult to end up in the top 53 applications. The distribution also reflects how PCs are configured by their vendors; on a new PC, you would more likely find recent security software than a recent blockbuster game.

For confidentiality reasons we are not able to explicitly name these applications specifically other than to note that these are some of the most commonly used applications in each domain. Throughout this paper, we refer to applications in an anonymized way; Internet-1 through Internet-14, Files-1 through Files-5, etc. This serves to show the intensity and trends of reliability effects within and between application domains without indicating the exact product or the associated organization that produced it.

Again, note that these categories only refer to machines whose users opted to send usage data. We discuss such issues in our threats to validity in Section VII.

\subsection{Characterizing Systems with Features}

For each machine observed, we analyzed configuration information and reliability data for the first week after the first startup of the operating system after installation. That is, when a new machine was enlisted in the CEIP between January and March, 2011, we examined data collected from the machine for just the first week. We specifically investigated this first week, as the initial impression with a new system very much determines user satisfaction; if the user experience is negative, he or she may even return a new computer.

The data consisted of a set of features that describe several aspects of each system: hardware configuration, applications installed and used as well as application failures. For the purpose of our study and to allow a more natural comparison, we dichotomized all features into binary variables. For app usage and installation this was fairly straightforward. For continuous measurements such as the amount of RAM installed or processor speed, we split about the median (the mean was not used due to skew in the data, indicating that a nonparametric measure was appropriate).

1. Hardware features. This includes seven features, which were true if they were above the median, and false if not. Generally, "true" values indicate more powerful machines.

- \# processors. True if more than two processors.

- Processor speed. True if 2,262 MHz or more.

- \# logical drives. True if four or more logical drives.

- \# physical drives. True if two or more physical drives.

- Drive size. True if 227,273 MB or more total space (used or unused) on all drives.

- Memory size. True if 2,933 MB or more total memory.

- Video memory. True if more than $128 \mathrm{MB}$ video memory.

2. Applications installed. If an application was launched once or more in the one-week period, we considered it to be "installed". (Note that a "launch" of an application may also be the launch of an installer belonging to that application.)
3. Applications used. If an application was launched more than five times in the one-week period, we considered it to be "used". (If an application was used less than five times, this indicates that it was just installed or tried, but never used again.)

4. Application failures. If any of the binaries of the application terminated abnormally at least once in the one-week period, we considered the application "crashing", indicating lowered reliability.

\subsection{Logistic Regression}

In order to relate application failures to hardware features, and to the presence and usage of specific applications, we used logistic regressions to determine how the individual factors influence failure probability [6]. Logistic regression allows us to determine the relationship that one factor has with a dichotomous outcome when controlling for other factors that may also be related to the outcome.

In our case, this allows us to answer questions such as for example "How is AdventureWorks reliability related to SouthBridge Video usage when controlling for the effects of system memory constraints?' By including many factors in one logistic model, we can take a global view of the interplay between many factors in different categories such as processor speed, specific applications installed, and specific applications used. Each logistic model has the following components:

- Reliability variable. For each application we built logistic models for a reliability variable $z$, which has a value of 0 if the application crashed in the first week after OS startup and 1 otherwise.

- Binary factors. For each regression model, we use as independent variables a set of binary factors, $x_{1}, x_{2}, \ldots, x_{n}$, which represent hardware features, applications installed, and/or applications used.

- Regression coefficients. Given a set of observations $\left(z, x_{1}, \ldots\right.$, $x_{\mathrm{n}}$ ) from our data set, each observation corresponding to one user's experience, logistic regression computes the best fit regression coefficients $\beta_{0}, \beta_{1}, \ldots, \beta_{n}$ such that

$$
P(z)=\frac{1}{1+e^{-\left(\beta_{0}+\beta_{1} x_{1}+\beta_{2} x_{2}+\cdots+\beta_{n} x_{n}\right)}}
$$

models the probability that $z$ has a value of 1 , that the application encounters no crashes.

Each of the regression coefficients $\beta_{i}$ indicates the sign and strength of the influence of the factor $x_{\mathrm{i}}$ within the model. The model also indicates the statistical significance of the variables (the probability that the variable actually has no effect on application reliability). For the statistical analysis we accounted for multiple hypothesis testing and considered only coefficients that are statistically significant at $\mathrm{p}<0.05$ after adjusting p-values with Benjamini-Hochberg [7] correction.

Let us assume that $z$ was the reliability variable for an image manipulation program, and $x_{1}, x_{2}, x_{3}$ would indicate if three applications were installed: an antivirus program $\left(x_{1}\right)$, an office application $\left(x_{2}\right)$, and a compression utility $\left(x_{3}\right)$. Then, in a hypothetical regression model such as

$$
\text { Reliability }=\frac{1}{1+e^{-\left(0.9+0.41 x_{1}-0.5 x_{2}+0.1 x_{3}\right)}}
$$

the positive coefficients $\beta_{1}$ and $\beta_{3}$ would show that the presence of the antivirus program and compression utility increased the reliability of image application (and the antivirus application does so by a stronger amount than the compression utility), whereas the presence of the office application decreased reliability (and increased the likelihood of the image application experiencing failure). If none of the 
three is present (all $x_{\mathrm{i}}=0$ ), the expected likelihood of image application not failing would still be $1 /\left(1+e^{-0.9}\right)=0.71$.

In logistic regression models, the coefficient $\beta_{i}$ corresponds to the change in the log of the odds for a one unit change in factor $x_{\text {i }}$ given that all other factors remain fixed. The odds ratio between absence and presence of a factor $x_{1}$ can be computed by raising $e$ to the power of the logistic coefficient, that is $O R=e^{\beta_{i}}$. In order to focus on strong effects, we considered only coefficients with a corresponding odd ratio of greater or equal than $3 / 2$, i.e., $\beta_{i} \geq \ln 1.5=0.40546$, or an odd ratio less or equal than $2 / 3, \beta_{i} \leq \ln (2 / 3)=-0.40546$. In the hypothetical regression model above, we would only consider the antivirus program $\left(\beta_{l}=0.41\right)$ and the office application $\left(\beta_{l}=0.5\right)$ to have strong effects on reliability.

Since all factors in our logistic regression model are binary, the direction (positive or negative) and magnitude of the coefficients can be directly compared to each other.

\subsection{Influence Networks}

For each application we built logistic models to model if the application failed in the first week after OS startup based on hardware features and other applications installed. We then used the coefficients to construct influence networks (similar to Heckerman et al. [8]). Each hardware feature and application is represented as a node. We create an edge between two nodes $\mathrm{X}$ and $\mathrm{Y}$ (where $\mathrm{Y}$ is an application), if the coefficient $\beta$ for $\mathrm{X}$ in the reliability model for $\mathrm{Y}$ was

- statistically significant at $\mathrm{p}<0.05$ after adjusting for multiple hypothesis testing [7], and

- strong, that is $\geq 0.40546$ or $\leq-0.40546$, corresponding to an odds ratio of $3 / 2$ and $2 / 3$ respectively.

Each edge is annotated with its corresponding coefficient $\beta$. The polarity of an edge is the sign of the coefficient; it can either be positive or negative.

In the resulting graph, the degree of nodes is an indicator of influence: nodes with many outgoing edges have strong influence on other applications, while nodes with many incoming edges are strongly influenced by others. We quantify influence of factors (nodes) within the graph as follows:

- Strength. The strength of a group of factors is the total number of outgoing edges from the group (actual influence) normalized by the total number of edges that are possible (maximum possible influence).

- Positivity. The positivity of a group of factors is the percentage of outgoing edges from the group that have positive coefficients, that is $\beta_{i} \geq 0.40546$.

Let us explain these measures with a few examples. The factor Processor speed influenced 11 out of 53 applications in our experiments, resulting in a strength of $11 / 53=20.8 \%$. All coefficients were positive and correlated with an increase in the reliability of the applications, resulting in a positivity of $11 / 11=100 \%$. The factor Internet- 1 influenced 8 out of 52 applications, a strength of $19.6 \%$. (Note that here we have only 52 applications because we did not include the factor Internet- 1 in the model for Internet-1.) Out of the 9 coefficients 8 were positive, resulting in a positivity of $88.9 \%$.

We also compute strength and positivity between groups of applications, for example to characterize the influence between Internet and other Internet applications or Internet and Security applications. The examples above are for influence relations, but strength and positivity can be computed in a similar way for relationships of type influenced by: instead of outgoing edges, one would consider ingoing edges.

\subsection{The "Influence Factors" Analysis Pattern}

The analysis discussed in the previous section can be generalized to be used in other contexts to answer other questions and thus represents a pattern of data analysis.

\section{Name: Influence Factors}

Intent: Uncover the influence of a set of entities on an outcome for each of those entities.

Input: A series of observations/instances, such that each instance includes factors for each of the entities and an outcome for each entity.

Output: The influence relationships. Either in table form or visualized as a network of influences [8]

\section{Analysis Steps:}

1. For each entity, $e$, build a logistic regression model which includes one independent variable for each of the other entities. For building the model for $e$, only use the input instances that include the outcome for $e$.

2. Select a threshold for the minimum level of influence that is of interest.

3. For each entity, $e$, the influences on $e$ are determined by examining the model for $e$ from step 1. The model coefficients that are both statistically significant and with a magnitude larger than the threshold indicate the entities that influence the outcome for $e$.

4. Record the influences of each entity on each other entity from step 3 into a table or into a graph in which an edge from entity $e$ to entity $f$ exists iff the model for $e$ shows an influence from $f$.

\section{Discussion:}

The steps for this pattern and their use in this paper use logistic regression to identify influences. However, logistic regression is just a parameter in the analysis. Other machine learning methods such as decision trees could also be used as long as the method indicates the relationship between the factor for each entity and the outcome for the entity of interest.

In addition, to ease analysis and assure consistency, the factor for each entity should be a binary factor. In the case of this paper, we use the presence of an application as a factor. We suggest that other factors be dichotomized, such as categorizing values based on a threshold (e.g. we used the median as a threshold for hardware features).

\section{Example Contexts:}

This pattern can be used in any context in which there are relationships between entities and an outcome at the entity-level. Such relationships include use of libraries, dependence of packages, and cochange of files. Outcomes of interest might comprise defects, build failures, or security vulnerabilities (similar to Neuhaus's examination of RedHat Packages [9]). The entities can also represent developers, for example to examine the influence that communication with different developers has on an OSS participant becoming a core developer (similar to Bird's study of open source participant immigration [10]).

As a more concrete example, we could use this pattern to evaluate the effects of component source code changes on build breaks in other components. The following table shows a portion of the input data. Each instance indicates which of the three components were changed and also what component was built and what the outcome was. In this example, the analysis would indicate that when $\mathrm{A}$ is 


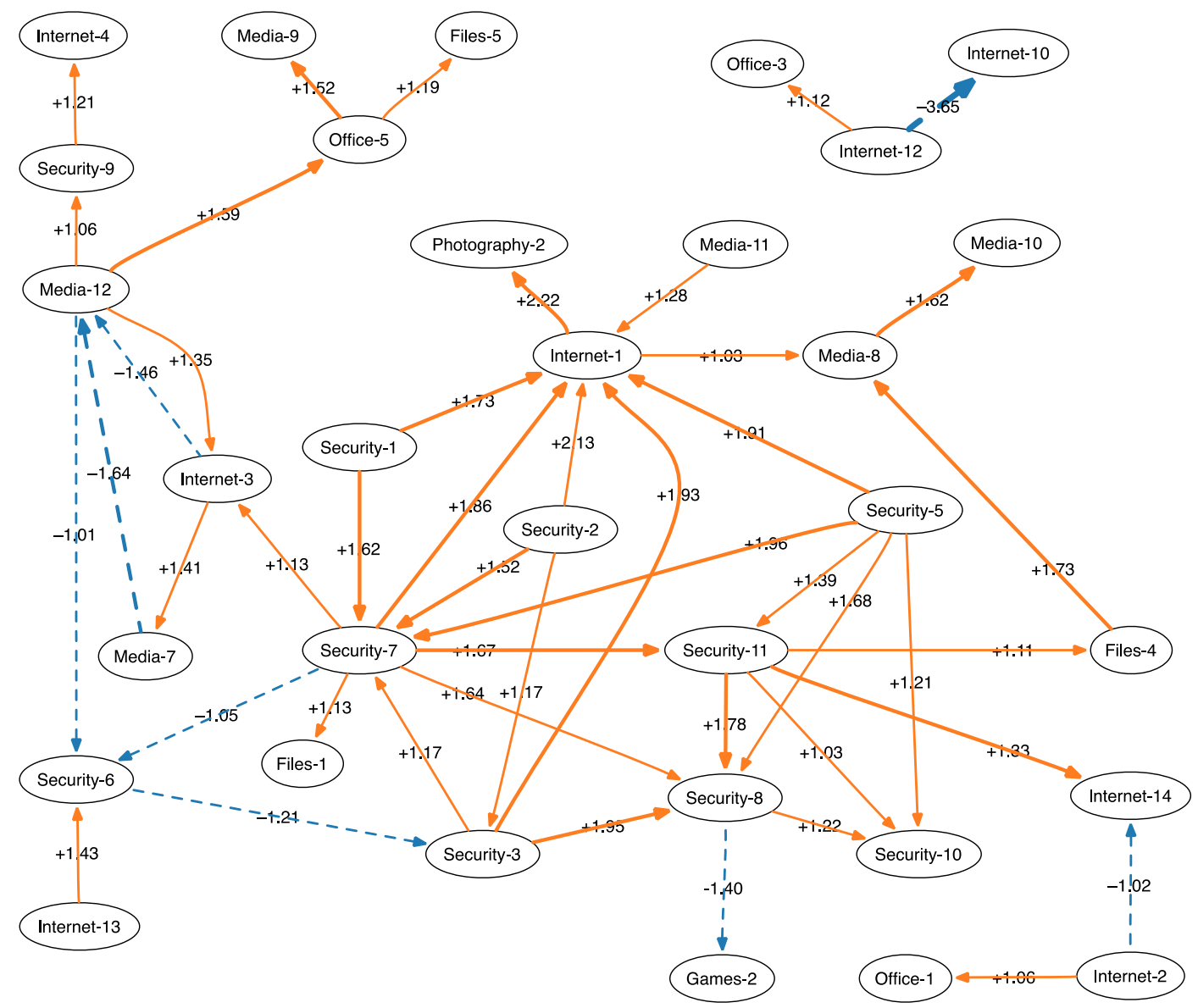

Figure 1. The strongest influence relationships between applications. Solid orange lines indicate that the source application has a positive relationship with reliability of the target application while dashed blue lines indicate a negative relationship. Regression coefficients for the models are also presented.

changed together with B, A fails to build. Thus B influences A's build outcome.

\begin{tabular}{ccc|cc}
\hline \multicolumn{3}{c|}{ Changed Component } & Built & Build \\
A & B & C & Component & Outcome \\
\hline 1 & 1 & 0 & A & Fail \\
1 & 0 & 1 & A & Pass \\
1 & 1 & 1 & A & Fail \\
0 & 1 & 1 & C & Pass \\
1 & 1 & 1 & B & Pass \\
\hline
\end{tabular}

\subsection{Association Rule Mining}

In addition to the identification of influence of individual factors (with logistic regression and influence networks), we identified the influence of factor combinations on application reliability. For this purpose, we employed association rule mining [11] to mine association rules of the form $\mathrm{X} \rightarrow \mathrm{Y}$, read as "if $\mathrm{X}$ occurs, then $\mathrm{Y}$ will occur", where $\mathrm{X}$ is a set of factors (such as an application installed) and $\mathrm{X}$ is called the antecedent and $\mathrm{Y}$ is the consequent.

For our study, we focus on rules in which $\mathrm{X}$ is a non-empty and nonsingleton set of features describing either hardware or application installation or application usage and Y describes the application reliability. Further, we only focus on rules that are statistically significant (i.e., support $>=0.1$ ), have high predictability (to protect the privacy of certain aspects of data, we cannot report the exact confidence threshold), and have high interest (i.e., lift $>=1.1)$. A rule $\mathrm{X} \rightarrow \mathrm{Y}$ with high lift suggests that the probability of $\mathrm{Y}$ increases (or decreases) by at least $10 \%$ when $\mathrm{X}$ occurs. To mine application-specific association rules, we consider for each application the application profiles of all machines on which the application was launched. Further, for each application, we mine three sets of rules by considering features describing hardware, application installation, and application usage separately. (We used the Apriori algorithm provided by the $\mathrm{R}$ arules library [12] to mine rules.)

\section{RELIABILITY FACTORS}

In this section, we show our results: How do individual features influence the reliability of individual applications? We start with hardware features.

\subsection{Hardware Features}

Our first hypothesis relates hardware features to application reliability:

H1. Less powerful hardware (processor speed, memory size...) is correlated with lower application reliability.

Our findings regarding the influence of hardware features are summarized in Table 1. The table lists for each feature the number of outgoing edges in the influence network with negative and positive polarity, followed by the Strength and Positivity scores for the feature. With regard to hardware factors, the number of processors, 
Table 1. Impact of hardware features on application reliability.

\begin{tabular}{llllrr}
\hline & & \multicolumn{3}{c}{ INFLUENC ON ReLIABILITY (53 apps) } \\
\cline { 2 - 6 } & FEATURE & - & + & Strength & Positivity \\
\hline above median & \# processors & 0 & 0 & $0.0 \%$ & \\
& Processor speed & 0 & 11 & $20.8 \%$ & $100.0 \%$ \\
& \# logical drives & 1 & 2 & $5.7 \%$ & $66.7 \%$ \\
& \# physical drives & 1 & 0 & $1.9 \%$ & $0.0 \%$ \\
& Drive size & 0 & 0 & $0.0 \%$ & \\
& Memory size & 0 & 6 & $11.3 \%$ & $100.0 \%$ \\
& Video memory & 0 & 0 & $0.0 \%$ & \\
\hline
\end{tabular}

drive size, and video memory size had no relationship with application reliability (strength of $0 \%$ ). While some coefficients were significant, none satisfied our thresholds of $+/-0.40546$. However, a higher-than-median processor speed is related to higher reliability of $20.8 \%$ of applications and a higher memory size is beneficial for $11.3 \%$ of the applications in our sample (both features have positivity of $100 \%)$. This confirms H1: A faster processor and more memory implies higher reliability.

A possible explanation can be that executing numerous programs concurrently on less powerful hardware leads to resource contention (for example memory), which in turn could lower application reliability. Unfortunately our data sources do not provide sufficient information to validate this explanation: in particular, we do not have access to third-party code.

\subsection{Installed Applications}

Let us now turn from hardware to software. Our hypothesis is that applications can impact each other's reliability:

H2. Usage of one application can influence the reliability of another application.

To investigate this hypothesis, we have plotted the strongest positive (plain arrows) and negative (dotted arrows) influences between applications in Figure 1. The figure only shows influences with a coefficient of less than -1 or greater than +1 . Note the relationship between Internet-12 and Internet-10 with a coefficient of -3.65 ; the corresponding odds ratio is $1 / 38.47\left(=e^{-3.65}\right)$. This indicates that in the presence of Internet-12, the reliability of the Internet-10 application is dramatically decreased. $\mathbf{H 2}$ is confirmed: Usage of applications influences the reliability of others.

\subsection{Application Categories}

Is it that just individual applications influence the reliability of others, or do similar applications share common traits? Our hypothesis is that

H3. There are categories of applications which tend to similarly impact applications (or be impacted by them).

To check this hypothesis, we have summarized our findings in a bubble heat map in Figure 2.

Here, each cell visualizes the strength and positivity of the influence of the category in the row on the category in the column. The size of the pie corresponds to the strength (the maximum strength in our experiments was 0.400 within Security applications). We have colorcoded cells to make it easier to identify patterns. Orange colors (light gray in black and white printouts) indicate positive, while blue colors (dark gray) indicate negative influence. The more orange, the higher the positivity. When the strength was zero (and positivity is undefined), the cell is left blank.

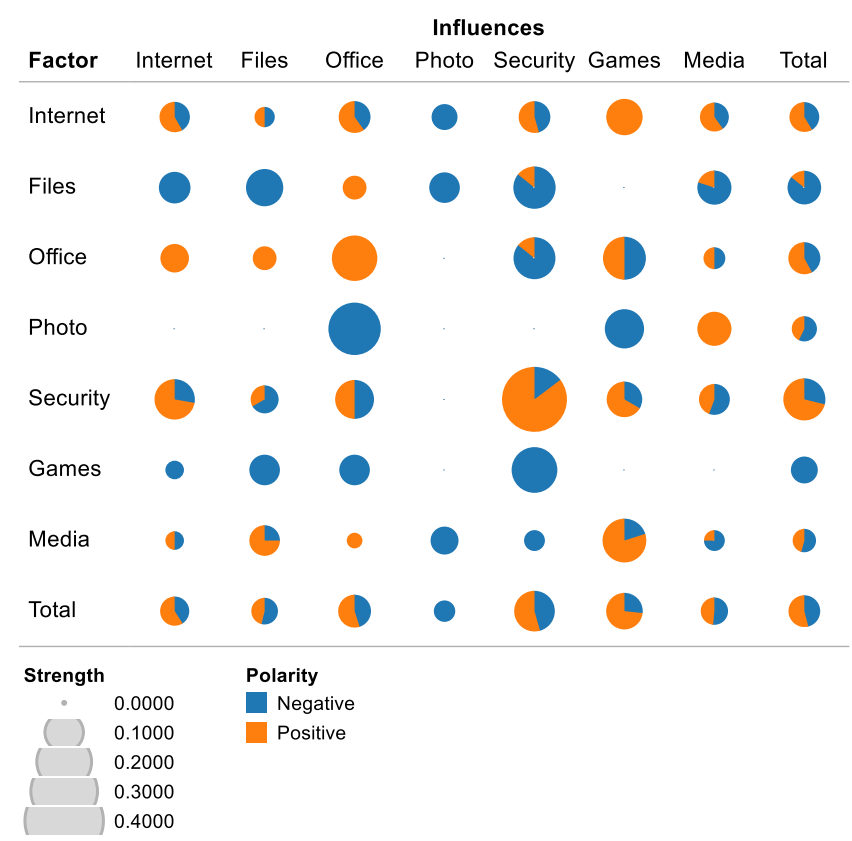

Figure 2. Impact of different categories on application reliability. Each row shows how an app category influences the reliability of other app categories. The width of a pie indicates the strength of the influence. The amount of orange (or light gray in black and white printouts) indicates the positivity.

By reading the figure by rows, one can see the influence of an application category on the reliability of other applications categories. For instance, the Photo category has negative influence on the reliability of Office and Games applications, but positive influence on the Media category. We also see that some categories (such as Files and Games) universally tend to decrease the reliability of others, supporting $\mathbf{H 3}$.

By reading the figure by column, one can see how an application category is influenced by others. We see, for instance, that the category Security is negatively influenced by all non-Security categories. H3 is thus confirmed: Some categories of applications impact others (and are impacted) in a similar way. Let us now discuss some findings in detail.

\subsubsection{File-sharing applications}

The Files row in Figure 2 tells a striking story: usage of file applications mostly correlated with lower reliability of other applications. Except for Office and Games applications the positivity is below $20 \%$. The total strength for all Files applications is $8.1 \%$ and the total positivity is $14.3 \%$. From a technical standpoint, this may come as a surprise: why should the installation of, say, a simple unarchiving program, affect the reliability of other programs?

One possible explanation is quite simple: it is not the file application itself that matters, it is how one uses it: to download content and programs from the Internet, sometimes from dubious sources - and it is these files which on average make other applications unreliable.

\section{In our study, usage of file and file-sharing programs is mostly correlated with lower application reliability}

\subsubsection{Office applications}

Office applications are slightly correlated with increased reliability. The total strength is $7.3 \%$ and the positivity is $57.9 \%$. There is a noticeable drop in positivity to $14.3 \%$ for security applications, which we will discuss below. 
Table 2. Impact of security applications on app reliability

\begin{tabular}{llrr}
\hline & & \multicolumn{2}{c}{$\begin{array}{c}\text { INFLUENCES OTHER APPS } \\
\text { (outgoing dependencies) }\end{array}$} \\
\cline { 2 - 4 } & FACTOR & Strength & Positivity \\
\hline application installed & Security-1 & $9.6 \%$ & $100.0 \%$ \\
& Security-2 & $13.5 \%$ & $100.0 \%$ \\
& Security-3 & $13.5 \%$ & $100.0 \%$ \\
& Security-4 & $3.8 \%$ & $50.0 \%$ \\
& Security-5 & $13.5 \%$ & $100.0 \%$ \\
& Security-6 & $7.8 \%$ & $50.0 \%$ \\
& Security-7 & $36.5 \%$ & $26.3 \%$ \\
& Security-8 & $7.7 \%$ & $100.0 \%$ \\
& Security-9 & $7.8 \%$ & $50.0 \%$ \\
& Security-10 & $1.9 \%$ & $100.0 \%$ \\
& Security-11 & $25.0 \%$ & $84.6 \%$ \\
\hline
\end{tabular}

We found usage of the office applications in our set to be slightly correlated with higher application reliability.

As with file-sharing applications, we believe that it is not the applications themselves, but the context in which they are being used that determines reliability. One might suspect that office applications are most often used in relatively well secure enterprise environment, and these environments contribute to improved application reliability. The office applications in our dataset are day-to-day applications used to view documents and perform minimal editing. Hence, it is indeed possible that office applications improve application reliability.

\subsubsection{Security applications}

Given the discussions on file sharing and office usage, one might assume that security applications generally improve reliability-after all, their purpose is to protect users from negative influences. Unfortunately, the picture is not that clear. Some applications (such as Internet-1) dramatically profit from the presence of any security application (see Figure 2). Also as Table 2 shows, six of the eleven security applications exclusively increase reliability when they do affect other applications (positivity of $100 \%$ ). However, not all security applications are beneficial to application reliability: Security7 is a security application whose presence correlates with almost universally reduced application reliability. It affects $36.5 \%$ of applications and for only $26.3 \%$ the influence was positive.

The category where security applications increase reliability the most is security applications (strength $30.9 \%$, positivity $85.3 \%$ ). Seven out of 11 security applications prevent other security applications from failing - an effect also visible in Figure 2. A possible explanation is that they prevent malware that would affect the next application in the command chain.

Security applications also increase the reliability of Internet (strength $11.8 \%$, positivity $72.2 \%$ ) and Games applications (strength $9.1 \%$, positivity $66.7 \%$ ). Overall, the total strength is $12.8 \%$ and the positivity is $71.2 \%$

In our study, security applications mostly increase the reliabil-
ity
of other applications.

Security applications may increase the reliability of other applications but they are negatively affected by almost every other application (see the Security column in Figure 2). With a few exceptions, the presence of other applications decreases reliability of security ap- plications. This could be due to several reasons. Security applications could be too restrictive (e.g. prohibit read access to certain registry keys), and this could cause application failures.

We found the reliability of security applications to be affected by most non-security applications.

\subsubsection{Games}

Looking at Figure 2, one might assume that makers of security applications must hate gamers: For all three games, installation correlates with increased chances of security applications failing (strength $15.2 \%$, positivity $0 \%$ ). This also holds for Internet, Files, and Office applications, which becomes significantly less reliable (positivity in all cases $0 \%$ ). Such effects may be due to specific usage profiles, such as a correlation between gaming and file sharing.

We found the three games in our set to be related to decreased reliability of other applications.

\subsection{Usage Frequency}

In our investigations thus far, we have not differentiated between whether an application was frequently used or just installed. This distinction makes a difference on how an application impacts others: Does this take place via some interference during installation? Or does mere execution of an application impact others? Our hypothesis is

H4. The more an application is used, the higher its impact on others.

To shed some light into this question, we built two additional regression models:

- Installation only (no further usage). The first model considers applications that were installed, but then hardly ever used. This category contained applications that were executed four times or less - typically, just the installation process, and 2-3 initial trial launches.

- Frequent usage. In the second model, we only looked at applications that were executed five times or more. (Again, the time period considered was the first week after the initial OS launch.)

Figure 3 shows the relationship of "installation-only" applications with reliability. For files, photography and game applications, their installation alone can be related to lower reliability of other applications (see the solid blue/dark gray bubbles, which indicate a positivity of $0 \%$ ).

For the other categories, the striking feature in Figure 3 is that only installing the applications but not using them has a mostly positive impact on reliability. Why would this be the case? Figure 4 gives a hint, showing the influence of "frequent usage" applications. What we see is a strong negative influence. In other words, installing (but not using) applications has a positive effect simply because of the lack of usage.

We thus concur that $\mathbf{H 4}$ is confirmed: The more an application is used, the greater the influence on reliability. However, this influence on reliability is generally negative.

\section{FEATURE COMBINATIONS}

Association rules based solely on hardware factors suggest that application reliability improved when the application was executed on powerful hardware. Further, the number of processors, number of logical drives, number of physical drives, memory size, and drive size occurred more frequently in the mined rules. This suggests that improvement in application reliability depends more on larger storage space and more powerful processing units. 
Of the 53 applications, we were able to generate rules for 25 applications based on application installation (I) and application usage (U) features. The set of all association mining rules is too large to present in this paper; we therefore present those rules that have the largest effect or are most interesting.

$\{$ I-Internet-6, I-Files-5 $\} \rightarrow$ DecreaseReliability-Media-4

$\{$ I-Security-8, I-Internet-9\} $\rightarrow$ IncreaseReliability-Media-4

$\{$ U-Media-1, U-Files-5 $\} \rightarrow$ DecreaseReliability-Media-4

$\{$ U-Office-2, U-Security-8\} $\rightarrow$ IncreaseReliability-Media-4

By construction, many feature combinations (i.e., antecedents) identified by association rules contained features (e.g. I-Files-5, U-Security-10) that already show a relationship with application reliability based on results from logistic regression. Besides such trivial feature combinations, association rules also identified feature combinations involving features that individually do not show a correlation with application reliability, e.g. $\{$ I-Office-2, I-Security-10 $\rightarrow$ IncreaseReliability-Files-2.

In addition, association rules identified the overall relationship of feature combinations with application reliability; specifically, when features involved in a combination had opposing relationships with application reliability when considered in isolation (as identified by logistic regression). For example, logistic regression suggested that the reliability of Internet-14 increases with the installation of Office2 and decreases with the installation of Files-5. However, the rule $\{$ IOffice-2, I-Files-5 $\} \rightarrow$ DecreaseReliability-Internet-14 suggested that the reliability of Internet-14 decreases when both Office- 2 and Files- 5 were installed. Further, in the 277 rules with application installation and usage features, features corresponding to 21 applications (out of 53 apps) appeared as part of antecedents.

Collectively, these observations suggest that combinations of features can affect application reliability even when features involved in a combination do not affect application reliability in isolation.

In our study, application reliability also depends on combinations

of features.

\section{DISCUSSION}

\subsection{How can applications affect each other?}

The first and foremost question is: Why and how does an individual application affect another application after all? Shouldn't the operating system protect applications from influencing each other? In principle, yes. However, an operating system should also enable applications to cooperate with each other. Consider sharing resources: If two applications $\mathrm{A}$ and $\mathrm{B}$ share the same library $\mathrm{C}$, and installing $\mathrm{A}$ updates $\mathrm{C}$ to fix the latest security issues, such an update may well trigger a bug in $\mathrm{B}$ that was previously masked. A similar situation can occur with registry entries that are shared by multiple applications (this is by design in many cases, allowing applications to become aware of each other and interact effectively). On Windows, several applications come with their own drivers and kernel extensions; security applications, as discussed above, hook in deep into the system. A games application taking control of the video hardware may change display resolution or prevent other applications from accessing the display - situations that other applications must be resilient to.

In this study, we did not investigate individual interferences. First of all, we suffer from a lack of more detailed usage profiles: We simply do not know how specific applications are being used, except for launches and failures - and this lack of knowledge is probably a

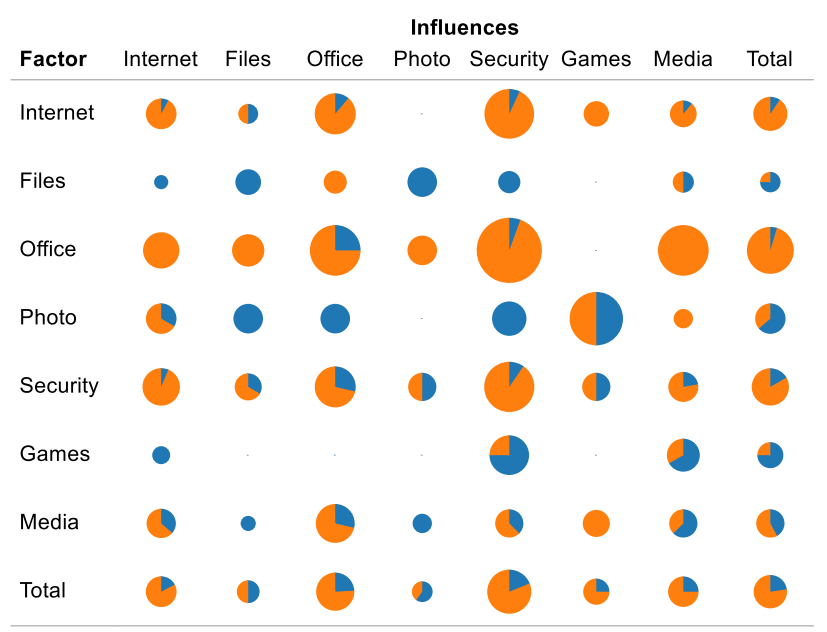

Figure 3. Impact on application reliability for installation only (no further usage)

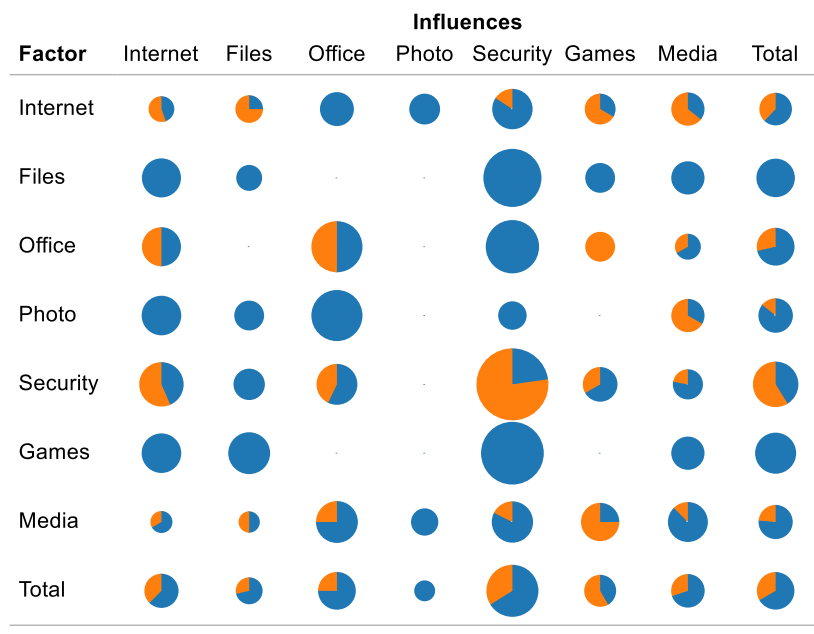

Figure 4. Impact on application reliability for installation and frequent usage

good thing. Likewise, we do not know how applications failed; we have no logs, stack traces, or like diagnostic information, nor can we debug third-party binaries.

In a well-designed and developed application, none of these issues should matter. But as the number of applications on a system grows, so do the possible negative influences.

\subsection{Implications of this work}

What we see in this study is that the reliability of an application can depend on factors that are not under control by application developers. This implies that assessing the reliability of an application in a single, well-defined context may produce an incomplete and inaccurate estimate of its real world reliability. This consequence affects the following fields:

- Testing is the most frequently used method to assess reliability. System testing is normally conducted in well-defined environments, such as an operating system installation out of the box. Our results imply that testing should place a special focus on real machines with different software configurations and usage profiles, in order to identify possible interferences from third-party applications and shared resources such as the Windows registry. Unfortunately, these additional demands on diversity further increase the complexity of system testing. 
- Application design must consider interdependences between applications more thoroughly. At one end, the design could reduce interdependences by keeping resources private. At the other end, the design could allow interdependences by sharing resources under well specified and honored contracts. How to specify such contracts at the system level is an open research issue.

- Operating system design will have to establish and enforce even stricter rules concerning interoperability of applications. This requires that such rules can be set and then checked for conformance; it may also mean that future applications be either much more limited or else much more explicit in the ways they integrate in the system.

- Reliability estimation and modeling has been an active area of research for many years [13], but to date has not included factors related to additional software installed and used on the same system in its models. Including such variables will likely lead to improved estimates and better statistical models.

- Further research is needed to explore ideas for better organized ecosystems with clear, enforceable, rules such that applications coexist better. How do we specify this? Who enforces the rules? Who should be "in charge" of the computer, vendors or users? All in all, there are plenty of challenges for systems and software research.

\subsection{Don't we know all this?}

One common misinterpretation of empirical studies is that nothing new is learned. However, such wisdom has rarely actually been shown to be true and is often quoted without scientific evidence. This paper provides such evidence: Most common wisdom is confirmed (e.g., "Less powerful hardware can lead to lower reliability") while some may come as a surprise (e.g., "The reliability of security applications is affected by most non-security applications").

Another frequent misconception is that empirical research within one context (in this case, applications running on Windows) is not good enough, provides little value for the academic community, and does not contribute to scientific development. Historical evidence shows otherwise. Flyvbjerg provides several examples of individual cases that contributed to discovery in physics, economics, and social science [14]. Beveridge observed for social sciences: "More discoveries have arisen from intense observation than from statistics applied to large groups" (as quoted in Kuper and Kuper [15], page 95). Please note that this should not be interpreted as a criticism of research that focuses on large samples or entire populations. For the development of an empirical body of knowledge as championed by Basili [16], both types of research are essential.

\subsection{Threats to Validity}

As with any empirical study, there are a number of threats to validity that must be discussed so that consumers of the study are aware of any caveats and are not misled.

Some applications are more frequently installed than others. As we have shown, there clearly are many relationships between the installation or usage of one application and the reliability of another. The more diverse the application profiles of the machines that an application is installed on, the more accurate the picture is. For applications that are not installed on as many machines as others, we may not identify relationships. Concretely, if application A is never installed on the same machine as application B, we will not be able to determine if there are reliability effects between the two. The same is true of hardware effects, if application $\mathrm{C}$ is only installed on machines with faster processors, then we are unable to determine the effect of processor speed on the reliability of $\mathrm{C}$. This threat is mitigated to some degree by the conjecture that if these factors never (or rarely) occurred in our data set, then even if there are reliability effects between them, they would not affect many users, since few users actually have both on their systems.

The previous conjecture holds if our sample is representative of the actual population of Windows users throughout the world. Our dataset consisted of close to 372,000 observations, the size of samples specific to each application ranged from 1,500 to 200,000 with a median of 15,000 . While this is a reasonable sample size, it is still only a small slice of total usage and it may not be representative of the actual population of Windows users. This threat could be mitigated by repeating the experiment with different data sets from the same population and averaging the results from these experiments.

Certain combinations of applications may more frequently occur on machines that are already unstable. For example, one common practice of users who are considered "hard core gamers" is a technique referred to as "overclocking" [17]. This entails increasing the processor speed beyond its specifications and can cause the machine to become unstable, leading to processor errors and application or system crashes. Such practices would occur more frequently on machines with games (especially CPU intensive games) installed than those without, which could lead to the conclusion that a particular game affects reliability when in fact the usage of the game is a proxy for user behavior that leads to decreased reliability.

Similarly, the presence of some applications may be indicative of other applications that were not prevalent enough to be included in our study. For instance, the use of file sharing apps may lead to installation of low quality software from less reputable sources. The presence of the application may act as a proxy for some unobserved factor; in this case, the presence of additional software that decreases the reliability of other apps.

The data corresponding to less powerful hardware may stem from older machines. Consequently, system age (both in terms of hardware and software) could be a latent factor that affects application reliability, e.g. low reliability of an old hard drive could be the cause of low application reliability and not the small size of the hard drive. We have not accounted for such latent factors as we do not have the data for system age.

\section{RELATED WORK}

Ours is by no means the first to examine software reliability; it has been studied from various viewpoints and for various reasons for decades [18] [19], and a vibrant research community has developed around the topic [20]. Empirical approaches to measuring software reliability hearken back to John Musa's data collection work in the 1970s [21].

Traditional software reliability research has focused mostly on intrinsic factors of the software. For example, Fosdick and Osterweil provide a survey of the ways that dataflow methods can be applied to software reliability [22]. Littlewood built models of reliability based on the modular structure of a piece of software and the transfer of control between modules [23]. Chen et al. found that the method of testing software - random test selection, structural test selection, or coverage based test selection - has a dramatic effect on software reliability estimates [24]. Goseva-Popstojanova et al. empirically evaluated architecture based software reliability modeling on GCC, an open source compiler, and found that such reliability modeling can be quite accurate, provided that enough data has been collected and the decomposition of the system into components can be determined [25]. These and similar software reliability approaches and studies focus on the software itself as the source of the reliability 
problem, while we examine an opposite, but complementary, view that extrinsic factors are also critical and should not be ignored when considering reliability.

Reliability has been empirically examined from a hardware perspective at scale. In a recent study of a million PCs, Nightingale et al. presented the first large scale hardware failure rate analysis [26]. They were able to identify hardware factors that affected system reliability both positively (underclocking and laptop use) and negatively (high CPU utilization and prior history of crashing). Our work is complementary in that our study comprises a large data set of users and identifies factors (both software and hardware) associated with application reliability.

The CEIP data has also been used to study reliability previously. Li et al. examined factors that led to system unreliability, quantified by the total number of system crashes and application hangs and crashes (irrespective of which application), between pre- and post-release of Windows [27]. They found that factors strongly related to system reliability included whether the OS was pre-installed by an OEM, 32 vs. 64 bit versions of Windows, and locale. Further, they found that these usage characteristics were markedly different between the Beta and Release of Windows 7 . We study software reliability at a more fine grained level by paying attention to individual applications. The goal of our work is not to single out specific applications as reliability factors, but rather to show that application reliability is affected by the presence of other applications.

\section{CONCLUSION}

On a real system, application reliability is affected by its environment, with hardware and usage of other applications being significant factors. Despite our best efforts, the reliability of our applications will thus be determined by factors that are not under our control. To put it with Sartre: L'enfer, c'est les autres - hell is other people.

The major consequence of this work is that assessing the reliability of an application in a single, well-defined context may produce an incomplete and inaccurate estimate of its reliability in the real world. This consequence affects many fields such as testing and verification, application design, operating system design, and reliability estimation and modeling.

In the real world, a society is the aggregate of people living together in a more or less ordered community. The applications and services on a computer form such a society as well, where the applications subject themselves to the given order, and where the operating system acts as the ordering force. As programmers, we would like to keep our freedom to have our programs do as they (and we) please. Future application societies, however, will have to enforce that one application's freedom ends where another begins - such that the interferences shown in this paper will be a thing of the past.

Acknowledgements. We thank the Windows team for their support in acquiring and interpreting the CEIP data. Brendan Murphy, Sriram Rajamani, Vince Orgovan, Thomas Ball, Juan Pablo Galeotti, Eva May, and Wolfram Schulte provided valuable feedback on earlier revisions of this paper. Andreas Zeller was a visiting researcher at Microsoft Research when this work was carried out. Venkatesh-Prasad Ranganath was a researcher at Microsoft Research, India, when this work was carried out.

\section{REFERENCES}

[1] Programs Which May Interfere with Steam. 2011. https://support.steampowered.com/kb_article.php?ref=9828-SFLZ9289.

[2] List of applications incompatible with Kaspersky Anti-Virus 2012. 2011. http://support.kaspersky.com/faq/?qid=20828435.
[3] Windows Customer Experience Improvement Program. 2011. http://technet.microsoft.com/en-us/library/ee126127(WS.10).aspx.

[4] Fleiss, J.L. Measuring nominal scale agreement among many raters. Psychological Bulletin, 76 (1971), 378.

[5] Landis, J.R. and Koch, G.G. The measurement of observer agreement for categorical data. Biometrics (1977), 159--174.

[6] Dowdy, S., Wearden, S., and Chilko, D.M. Statistics for research. Wiley New York, 1983

[7] Benjamini, Y. and Hochberg, Y. Controlling the false discovery rate: a practical and powerful approach to multiple testing. Journal of the Royal Statistical Society. Series B (Methodological), 57, 1 (1995), 289300.

[8] Heckerman, D., Chickering, D., Meek, C., Rounthwaite, R., and Kadie, C. Dependency Networks for Collaborative Filtering and Data Visualization. In Proceedings of the Sixteenth Conference Annual Conference on Uncertainty in Artificial Intelligence (UAI-00) (2000), 264-273.

[9] Neuhaus, S. and Zimmermann, T. The Beauty and the Beast: Vulnerabilities in Red Hat's Packages. In Proceedings of the USENIX Annual Technical Conference (2009).

[10] Bird, C., Gourley, A., Devanbu, P., Swaminathan, A., and Hsu, G. Open Borders? Immigration in Open Source Projects. In Proceedings of the 4th International Workshop on Mining Software Repositories (2007).

[11] Agrawal, R. and Srikant, R. Fast Algorithms for Mining Association Rules in Large Databases. In VLDB '94: Proceedings of the 20th International Conference on Very Large Data Bases (1994), 487-499.

[12] ARules. http://r-forge.r-project.org/projects/arules/.

[13] Farr, W. Software reliability modeling survey. In International Symposium on Software Reliability Engineering (1996).

[14] Flyvbjerg, B. Five misunderstandings about case-study research. Qualitative inquiry, 12, 2 (2006), 219-245.

[15] Kuper, A. and Kuper, J. The Social Science Encyclopedia. Routledge, 1985.

[16] Basili, V.R., Shull, F., and Lanubile, F. Building knowledge through families of experiments. IEEE Trans. Software Eng., 25, 4 (1999).

[17] Wanniner, S. and Richmond, R. The Book of Overclocking. No Starch Press, 2003.

[18] Gray, J. Why do computers stop and what can be done about it. In Symposium on reliability in distributed software and database systems (1986).

[19] Musa, J. Software Reliability Engineering. Osborne/McGraw-Hill, 1998

[20] Marathe, M. and Cukier, M. In Proceedings of the IEEE 21st International Symposium on Software Reliability (2010).

[21] Musa, J.D. A Theory of Software Reliability and Its Application. IEEE Transactions on Software Engineering, 1, 3 (1975), 312--327.

[22] Fosdick, L.D. and Osterweil, L.J. Data Flow Analysis in Software Reliability. ACM Computing Surveys, 8, 3 (1976), 305--330.

[23] Littlewood, B. Software Reliability Model for Modular Program Structure. IEEE Transactions on Reliability, R-28, 3 (1979), 241--246.

[24] Chen, M.-H., Mathur, A.P., and Rego, V.J. Effect of testing techniques on software reliability estimates obtained using a time-domain model. IEEE Transactions on Reliability, 44, 1 (1995), 97--103.

[25] Goseva-Popstojanova, K., Hamill, M., and Perugupalli, R. Large Empirical Case Study of Architecture-Based Software Reliability. In Intl. Symposium on Software Reliability Engineering (2005), 43-52.

[26] Nightingale, E.B., Douceur, J.R., and Orgovan, V. Cycles, cells and platters: an empirical analysisof hardware failures on a million consumer PCs. In EuroSys (2011).

[27] Li, P.L., Kivett, R., Zhan, Z., Jeon, S.-e., Nagappan, N., Murphy, B., and Ko, A.J. Characterizing the differences between pre and post release versions of software. In International Conference on Software Engineering (2011). 